\title{
Dilemas de la inclusión y discapacidad en la educación superior
}

\author{
Judith PÉrez CASTro*
}

En este artículo discutimos tres formulaciones del dilema de la inclusión de las personas con discapacidad en la educación superior: la primera se refiere al mérito, la segunda a la vocación y la tercera a los ajustes. Partimos de la idea de que los dilemas son construcciones que los sujetos hacemos a partir de un determinado problema y que, en el caso de los estudiantes con discapacidad, se originan por la complejidad que conlleva lograr un equilibrio entre el trato diferencial y similar hacia estas personas. A ello contribuye el hecho de que las instituciones educativas tienen normas, prácticas y condiciones poco incluyentes, lo que genera tensiones entre los diferentes actores educativos. Finalmente, si bien los dilemas, por definición, no tienen una solución definitiva, consideramos que abren espacios para reflexionar sobre el quehacer de las instituciones educativas, el trabajo docente, los contenidos curriculares y nuestras ideas sobre la diferencia.

The present article discusses three ways to formulate the dilemma of the inclusion of people with disabilities in higher education: the first refers to merit, the second to vocation and the third to adjustments. We start from the idea that dilemmas are constructions that the subjects make based on a certain problem and that, in the case of students with disabilities, they originate from the complexity involved in achieving a balance between differential and similar treatment. The fact that educational institutions have not very inclusive norms, practices and conditions contributes to this, which generates tensions between the different educational actors. Finally, although dilemmas by definition do not have a definitive solution, we consider that they open spaces to reflect on the work of educational institutions, teaching work, curricular content and our preconceptions about difference.
Palabras clave

Inclusión educativa

Educación superior

Discapacidad

Profesores

Estudiantes

Keywords

Educational inclusion

Higher education

Disability

Teachers

Students

Recepción: 2 de octubre de 2020 | Aceptación: 4 de marzo de 2021

DOI: https://doi.org/10.22201/iisue.24486167e.2022.175.60179

* Investigadora del Instituto de Investigaciones sobre la Universidad y la Educación (IISUE) de la Universidad Nacional Autónoma de México (UNAM) (México). Doctora en Ciencia Social con especialidad en Sociología. Líneas de investigación: equidad e inclusión educativa; políticas educativas; ética y valores profesionales. Publicaciones recientes: (2021), "Condiciones para la docencia inclusiva: análisis desde las barreras y los facilitadores", Revista Iberoamericana de Educación Superior, vol. 12, núm. 33, pp. 138-157; (2021), "La pandemia como factor de exclusión de los estudiantes con discapacidad en la educación superior", Educación Superior y Sociedad, vol. 2, núm. 33, pp.470-495.CE: pkjudith33@yahoo.com.mx 


\section{INTRODUCCIÓN ${ }^{1}$}

La inclusión se ha convertido en un tema que permea gran parte de la discusión académica, así como el quehacer de los actores educativos. Cada vez hay un mayor consenso en que la educación es un derecho fundamental y habilitador que debe garantizarse a todas las personas, en igualdad de condiciones, independientemente de su situación social o condición de vida (UNESCO, 2017). Sin embargo, este compromiso ha generado nuevas presiones e incrementado las ya existentes en las instituciones educativas, ante las demandas de una población estudiantil más diversa, con intereses específicos y con puntos de partida diferenciados.

Este fenómeno no es nuevo; la igualdad de oportunidades ha sido una lucha emprendida desde hace mucho tiempo ante el predominio de ideas y prácticas excluyentes en los sistemas educativos (Tomasevki, 2004) y aún hoy, que la cobertura se ha ampliado a nivel mundial, particularmente en la educación básica, no todos tienen acceso a la misma escuela y, por lo tanto, a la misma competencia (Dubet, 2005). La diferencia con el enfoque de la inclusión educativa es que el énfasis se ha puesto tanto en el reconocimiento de la diversidad humana como en la eliminación de las barreras institucionales que limitan la participación y oportunidades de aprendizaje de niños, niñas, jóvenes y adultos, primordialmente de aquéllos que están en situaciones excepcionales o de vulnerabilidad (UNESCO, 2017).

En este contexto, en el que la diversidad se ha asumido como un valor importante, se refuerzan los dilemas que enfrentan las instituciones en su empeño por desarrollar condiciones que permitan a todos ejercer su derecho a la educación con igualdad y, al mismo tiempo, reconocer sus diferencias. Uno de los trabajos pioneros sobre el tema es el de Harry Judge (1981), quien distingue cinco grandes disyuntivas: 1) la utilidad económica de la educación versus la realización personal y la armonía social; 2) la justicia de los métodos de selección contra su eficiencia y confiabilidad; 3) lo común en oposición a lo diverso de los contenidos curriculares; 4) la centralización de las decisiones educativas frente a la autonomía institucional; y 5) la diversificación versus la especialización del trabajo docente.

A estos dilemas, aún vigentes en la actualidad, se ha sumado el reto de construir instituciones educativas incluyentes $\mathrm{y}$ atentas a las diferencias de los estudiantes en donde, al mismo tiempo, se brinde un "piso común" en el que todos aprendan, participen y se sientan reconocidos. Nuestro objetivo en este artículo es analizar algunos de los dilemas que surgen en el proceso de inclusión de los estudiantes con discapacidad en la educación superior. Para ello, analizamos algunas ideas en torno a la relación entre estigma y diferencia, para después adentrarnos en los desarrollos que autores como Dyson y Millward, Artiles y Norwich han elaborado en torno al dilema de la diferencia. Posteriormente, exponemos tres disyuntivas que ha generado la inclusión de las personas con discapacidad en la educación superior: el dilema del mérito, el de la vocación y el de los ajustes. Para cerrar, hacemos algunas consideraciones generales sobre los aspectos aquí tratados.

\section{LA DIFERENCIA COMO \\ DILEMA EN LA EDUCACIÓN}

Los dilemas son situaciones problemáticas que conllevan la toma de decisiones frente a una serie de alternativas en las que se ven comprometidos uno o varios principios éticos (Allen, 2012). De acuerdo con Banks y Williams (2005), los dilemas producen incomodidad e incertidumbre porque hay evidencias a favor y en contra de los diferentes cursos de acción, lo que hace que su resolución no sea definitiva. Es decir, siempre existirá la duda

\footnotetext{
${ }^{1}$ Este artículo es producto una investigación financiada por el Programa de Apoyos a Proyectos de Investigación e Innovación Tecnológica (PAPIT), de la Universidad Nacional Autónoma de México.
} 
respecto a la idoneidad de la decisión asumida, porque muchas veces lo más que podemos hacer, dependiendo de la información, recursos y situación con los que contemos, es escoger la alternativa menos desagradable o dañina para nosotros y los demás involucrados. "Una situación o evento no es por sí mismo un dilema, pero puede ser construido como tal por ciertas personas" (Banks y Williams, 2005: 1011) (traducción propia).

Específicamente sobre el dilema de la diferencia, sus orígenes se remiten al esfuerzo que hacemos para darle sentido a las cosas, personas y acontecimientos de la vida cotidiana, ya que, si bien la mayoría de ellos tiene significados socialmente construidos que se mantienen estables por un largo tiempo, los sujetos solemos renegociarlos en el transcurso de nuestros encuentros sociales (Coulon, 1995; Minow, 1990). En el intercambio diario entre personas, el contexto social brinda diferentes elementos para que los participantes cumplan con las pautas a las que estamos acostumbrados; sin embargo, esta aparente normalidad se rompe cuando nos percatamos de que el otro que está frente a mí tiene algo que lo distingue y que no corresponde a los rasgos socialmente valorados. "Un atributo de esa naturaleza es un estigma, en especial cuando él produce en los demás, a modo de efecto, un descrédito amplio... Esto constituye una discrepancia entre la identidad social virtual y la real" (Goffman, 2001: 12-13).

El problema del estigma no es la diferencia por sí misma, sino los efectos que tiene en las expectativas sociales, ya que usualmente suponemos que quien pertenece a una categoría social determinada no sólo deberá apoyar las normas en las que ésta se sustenta, sino que también las deberá acatar (Goffman, 2001). Asimismo, la diferencia no tiene mayor trascendencia cuando la interacción se da entre iguales; las dificultades surgen en los encuentros sociales mixtos, cuando participa una persona visiblemente diferente a las demás o con un estigma desacreditado. En este tipo de interacción, los participantes sienten que pierden el control; "podemos sentir que, si manifestamos un interés sensible y directo por su condición, nos estamos extralimitando, y que, sin embargo, si olvidamos verdaderamente su defecto podemos llegar a tener con él exigencias imposibles..." (Goffman, 2001: 30).

A partir de estas ideas de Goffman, Minow (1990) construye el dilema de la diferencia para analizar el sistema jurídico estadounidense y sostiene que el problema fundamental que aquélla plantea es que históricamente la hemos entendido como algo negativo, pero, al mismo tiempo, hemos tratado de repararla o disimularla. La paradoja es:

¿Cuándo tratar a las personas de manera diferente se convierte en una base que enfatiza sus diferencias, las estigmatiza y las obstaculiza? Y ¿cuándo tratarlas de la misma manera se vuelve insensible a sus diferencias, lo que muy probablemente las estigmatiza u obstaculiza de nueva cuenta? (Minow, 1990: 20) (traducción propia).

La respuesta no es sencilla porque, sin importar el camino que tomemos, el estigma de la diferencia tiende a reforzarse al ponerle atención o al ignorarla. Y esto ocurre en mayor o menor medida con todo tipo de diferencias: lingüísticas, étnicas, de género, de capacidad o religiosas.

En la educación, la diferencia se convirtió en un dilema a partir de la ampliación de oportunidades para los denominados grupos minoritarios (Lowe, 1999): mujeres, afrodescendientes, indígenas, migrantes $y$, más recientemente, personas con discapacidad. Hasta mediados del siglo XX, muchos de ellos eran excluidos de los sistemas educativos o canalizados a modalidades segregadas, sin embargo, las reformas integradoras emprendidas en distintos países - y atizadas, en buena medida, por los movimientos a favor de los derechos de las minorías - incrementaron sus posibilidades de matricularse en la educación regular. No obstante, en lugar de favorecer al 
reconocimiento de sus diferencias, esto fortaleció la idea de que se tenía que hacer un mayor esfuerzo para que se adecuaran a las normas y valores de la cultura dominante (Parrilla, 2002).

De este modo, el dilema de la diferencia en educación es resultado de la disyuntiva que enfrentan las instituciones entre reconocer o no las diferencias de los alumnos y, sobre todo, la forma de responder a ellas, dado que ambas opciones entrañan serias consecuencias (Norwich, 2013). Como en cualquier otro dilema, en el de la diferencia intervienen las ideas, principios y valores de los sujetos, así como las condiciones y recursos de los que disponen; es decir, se trata de construcciones que hacen los propios sujetos. Esto no significa que no sean reales; de hecho, los dilemas tienen un núcleo problemático bastante real $y$, aunque no necesariamente se puede llegar a una solución definitiva, brindan la posibilidad de reflexionar sobre las condiciones institucionales, así como sobre el papel de los actores educativos en el mantenimiento y superación de tales disyuntivas.

En este artículo abordamos una de las dimensiones del dilema de la diferencia, la que remite a la inclusión educativa de las personas con discapacidad. A continuación, presentamos tres enfoques desde donde se ha analizado esta problemática.

\section{El dilema de la inclusión desde la dimensión organizacional}

La primera perspectiva que queremos tratar es la de Allan Dyson y Allan Millward, los cuales parten de la idea de que los sistemas educativos han respondido de manera poco eficiente ante las diferencias; sostienen que la educación inclusiva ha sido sólo una de las diferentes vías que se han ensayado, pero de ninguna manera puede considerarse la solución definitiva a los problemas que enfrentan las instituciones.

Los autores sitúan la mirada en el nivel organizacional y afirman que el meollo de la contradicción fundamental consiste en que los sistemas fueron diseñados para brindar educación a la población de cada territorio nacional. Esta educación posee ciertos rasgos comunes que han sido definidos socialmente en términos de contenidos y habilidades, sin embargo, los estudiantes son personas con intereses y expectativas particulares, aptitudes y limitaciones distintivas y formas de aprendizaje propias que los hacen muy diferentes entre sí. De este modo,

...junto con el requisito para que el sistema educativo haga algo reconociblemente similar para todos los estudiantes, existe un requisito igualyopuesto paraquehaga algodiferentecon cada alumno. De ello se desprende que los sistemas necesariamente se vean forzados en distintas direcciones por estas demandas (Dyson y Millward, 2000: 161) (traducción propia).

Las contradicciones surgen a partir de las decisiones que toman las instituciones educativas, y más concretamente sus actores, en un esfuerzo por resolver el dilema de la diferencia (Dyson, 2001). Es decir, como en cualquier organización, éstas funcionan a partir de normas y procesos estandarizados que les proveen estabilidad y, al mismo tiempo, les dan cierto margen para responder a la variabilidad de sus usuarios. Pero este rango tiene sus límites, de tal manera que siempre tratan de que los usuarios adecúen sus necesidades al ámbito de su especialización y, cuando esto no es posible, los empujan a salirse (Skrtic, 1991).

Algo similar sucede con los estudiantes con discapacidad: aquéllos cuyas necesidades están más allá de los márgenes de acción institucionales suelen ser vistos como problemáticos y son apartados de la dinámica escolar, o bien, canalizados a la educación especial. Una vez más, esto produce situaciones dilemáticas, porque si los alumnos son incluidos en las mismas escuelas, en las mismas aulas y con currículos comunes, sus diferencias tienden a convertirse en tensiones para los docentes y directivos, mientras que, si son atendidos en 
establecimientos especiales, se refuerzan sus diferencias debido a la limitada capacidad de estas escuelas para reconocer lo común de la humanidad (Dyson, 2001).

Además, como ocurre en toda organización, las instituciones educativas están sujetas a los intereses individuales y de grupo, y esto incide en la definición que los actores educativos hacen de los dilemas, en las medidas que se tomen para su resolución y en los conflictos que se generen a partir de ellos (Dyson y Millward, 2000). En ese sentido, las acciones que se tomen frente a los dilemas, además de estar en función de las normas, prácticas y recursos institucionales, también lo estarán de las metas y activos de los actores educativos y de los grupos a los que pertenecen.

El punto es que el dilema de la diferencia no tiene una solución definitiva y los acuerdos a los que se llegue para manejarlos frecuentemente conllevarán beneficios para la comunidad escolar, pero también costos que no siempre son fáciles de asumir. En todo caso, siempre se abren oportunidades para el cambio, especialmente en la cultura y los valores institucionales.

En donde el cambio sea mal manejado... los conflictos se exacerbarán, los dilemas se agudizarán y la solución elegida no se realizará. Por otro lado, si el cambio se maneja hábilmente, aunque ni los dilemas ni la contradicción subyacente desaparezcan, puede surgir una nueva solución y concretarse con un grado relativamente alto de estabilidad (Dyson y Millward, 2000: 167) (traducción propia).

Finalmente, los autores advierten que es necesario que los tomadores de decisiones, y nosotros agregaríamos a los docentes, evalúen cuidadosamente las opciones que toman ante el dilema de la diferencia en relación con las alternativas disponibles y aquéllas que han sido rechazadas. Asimismo, es importante que traten de entender tanto al dilema como a las contradicciones que le dieron origen y que recuerden que la solución a la que se ha llegado no es concluyente, sino el resultado de un tiempo, lugar y circunstancias determinadas.

\section{La dualidad diferencia-representatividad}

En este apartado nos interesa discutir los aportes de Alfredo Artiles (1998), cuyo trabajo se sitúa en la disyuntiva entre la representatividad y el reconocimiento de las diferencias de aquellos colectivos que han sido excluidos o segregados de las instituciones educativas. Su planteamiento inicial es que, para comprender la representación desproporcionada de ciertas minorías en la educación especial, es necesario examinar los supuestos que socialmente hemos construido sobre la diferencia y que están en la base de los dilemas educativos. Para ello, retoma el trabajo de Martha Minow (1990), quien sostiene que, a lo largo de la historia, las sociedades han desarrollado distintas formas para tratar a los diferentes, a quienes les ha atribuido categorías que los estigmatizan, pero, sobre todo, determinan sus oportunidades de ser incluidos o excluidos de la vida política, económica y social.

De este modo, la sociedad ha asumido a la diferencia como algo problemático y, por extensión, las personas que no se ajustan a lo socialmente establecido son valoradas de la misma manera. De acuerdo con Artiles (1998), esta idea está sustentada en tres pilares: el pensamiento deficitario sobre las diferencias culturales; la pretensión de neutralidad del conocimiento científico; y el supuesto de que el punto de vista de los sujetos pertenecientes a los grupos minoritarios no siempre es relevante. Particularmente sobre la población con discapacidad sostiene que, para empezar, los avances en la educación especial deben analizarse desde los procesos culturales y políticos que han surgido en el marco de las reformas educativas. También señala que es necesario reconocer los múltiples dilemas que acarrean tanto la inclusión de ciertos alumnos en la enseñanza regular como su sobrerrepresentación en la educación especial, sobre todo por sus 
alcances en el desarrollo de sistemas educativos justos; por ejemplo, el incremento de las personas con discapacidad plantea el reto de contar con propuestas teóricas y políticas públicas que permitan atender la diversidad cultural y las capacidades individuales de los estudiantes. Particularmente, a los docentes los enfrenta con la tarea de comprender el lugar que tiene la cultura en las ideas que históricamente se han elaborado sobre estos individuos (Artiles, 2003). Ésta no es una tarea fácil, porque implica que ellos, los maestros, cuestionen sus propias visiones sobre la diferencia, valoren críticamente los contenidos y métodos de enseñanza y transformen las prácticas que obstaculizan la participación de todos los alumnos.

Para Artiles (2003) el movimiento de la educación inclusiva invariablemente conducirá a la transformación de la educación especial. Dos de sus fortalezas han sido la promoción del sentido de comunidad para el logro de los aprendizajes y la defensa de que la atención a los estudiantes debe partir del conocimiento de sus necesidades, y no del diagnóstico de determinadas capacidades. El problema es que las expectativas hacia las personas de los grupos minoritarios tienden a ser bajas; se cree que no poseen los conocimientos, disposiciones y valores requeridos por las instituciones educativas y que lo mejor es enviarlos a la educación especial (Artiles, 2003). Es decir, una vez más, las diferencias son vistas desde la perspectiva deficitaria y, como corolario, los alumnos son clasificados desde sus carencias respecto de quienes cumplen con el "promedio". Sorprendentemente, muy pocas veces nos preguntamos el porqué de la sobrerrepresentación de ciertos grupos sociales en la educación especial. Más paradójico aún es que

“...a pesar de que el movimiento de educación inclusiva representa el surgimiento de voces empoderadas sobre los derechos de las personas con discapacidad y de mejores servicios educativos para ellas, ha permanecido en silencio sobre la difícil situación de los estudiantes minoritarios (Artiles, 2003: 177)

(traducción propia).

En este contexto, los dilemas surgen porque, en las instituciones educativas, la diversidad usualmente se relaciona con las diferencias en las habilidades de los educandos, lo cual puede ser un insumo relevante para los procesos de enseñanza, pero en los alumnos de grupos minoritarios esto tiende a asociarse con los rasgos negativos que históricamente se les han imputado. La educación especial, por su parte, puede complicar más la situación de las personas con discapacidad porque añade "otra capa de diferencia a las minorías raciales, restringe su acceso a programas y oportunidades educativas altamente valorados $y$ limita aún más sus resultados educativos a largo plazo" (Artiles, 2003: 176) (traducción propia). Es decir, termina por profundizar el problema que inicialmente buscaba resolver. A pesar de esto, Artiles es optimista y piensa que el debate en torno a la sobrerrepresentación es una coyuntura para mirarnos desde dentro y reflexionar críticamente sobre nuestros supuestos culturales.

\section{Los dilemas como dimensión \\ de la inclusión educativa}

A partir de los años noventa ha venido cobrando fuerza la idea de que necesitamos construir sistemas educativos que asuman la diversidad de la condición humana y que valoren positivamente las diferencias, ya sean sociales, culturales, funcionales, lingüísticas o de cualquier otro tipo. Pero, reconocer todo esto requiere de un equilibrio que no siempre es fácil de mantener. Éste es el punto de partida del trabajo de Brahm Norwich (2008a) respecto a lo que denomina el dilema de la inclusión de las personas con discapacidad. Para este autor, en la educación ha habido un desinterés generalizado por acercarse a los dilemas, lo cual se debe, en gran medida, a la incertidumbre que producen dado que nunca quedan totalmente resueltos. 
En el origen de los dilemas están las ideas que socialmente hemos construido sobre el "deber ser" de las instituciones y los actores educativos; particularmente, en el dilema de la diferencia se encuentra la tensión entre la perspectiva negativa, que se asocia con la minusvalía, el estatus inferior, el trato injusto y los servicios educativos de baja calidad; y la perspectiva positiva, en la que la diferencia se vincula con el reconocimiento de la individualidad, así como de las necesidades e intereses particulares (Norwich, 2008b). A esto se suman los problemas teóricos y prácticos que conlleva el enfoque de la educación inclusiva.

En la dimensión teórica, Norwich (2013) destaca tres dificultades. La primera tiene que ver con la difusión que la inclusión ha tenido a nivel internacional, lo que la ha convertido en un asunto bueno en sí mismo; sin embargo, esta visibilidad también ha diversificado su significado, de manera que por inclusión entendemos cada vez más cosas, desde el aprendizaje y la participación en la vida escolar, hasta cuestiones más encaminadas al logro de sociedades incluyentes. La segunda problemática es un efecto no calculado de la aspiración de una educación para todos, muchas veces sustentada en valores muy amplios o abstractos, que hace que las circunstancias específicas de las personas se desdibujen en el amplio mar de la diversidad. "La discapacidad se convierte en una de las distintas facetas de la diversidad, al igual que la etnicidad, el género, el aprendizaje de una segunda lengua y las desventajas socioeconómicas" (Norwich, 2013: 3) (traducción propia). En el otro extremo ocurre que la inclusión parece quedar anclada únicamente a la discapacidad, sin considerar otras formas de diversidad como la edad, el género o la etnia. La tercera dificultad teórica apunta al uso de categorías especializadas en los sistemas educativos. Aquí, el problema radica en determinar en qué circunstancias los componentes diferenciados del currículo y los enfoques educativos pueden ser excluyentes para los estudiantes y en qué otras contribuyen realmente a responder a sus necesidades (Norwich, 2013).

Con respecto a los problemas prácticos, Norwich (2014) señala que, a través del concepto de "barreras", el enfoque de la inclusión ayudó a situar la mirada sobre el origen de la discapacidad en el medio social, no obstante, a veces se nos olvida que la inclusión tiene sus límites. Es decir, aunque se removieran cada una de las barreras del contexto, no todos podrían ser plenamente incluidos, porque los factores que intervienen en la construcción de la discapacidad no sólo son externos e internos a las personas, sino que además tienen un rango de estabilidad y alteración, de manera que algunos pueden ser modificados o compensados y otros no.

A partir de estas reflexiones Norwich (2008a) identifica tres dilemas resultantes de la forma en que las instituciones educativas han abordado las diferencias: el dilema de identificación, el del currículo y el de ubicación: en el primero, la disyuntiva está en que cuando los estudiantes con dificultades son identificados como personas con discapacidad, suelen ser estigmatizados y tratados de manera diferente, pero cuando la discapacidad no se reconoce, entonces las posibilidades de asegurarles los apoyos educativos disminuyen. En el dilema del currículo la complejidad consiste en que cuando se proporcionan las mismas experiencias de aprendizaje para todos, incluyendo a los alumnos con discapacidad, existe el riesgo de que se reduzcan o incluso se les nieguen oportunidades de aprendizaje significativas, no obstante, cuando no se les ofrecen las mismas condiciones que a sus pares, el peligro es la segregación y, una vez más, coartar sus oportunidades de aprendizaje (Norwich, 2008a).

El tercer dilema, referido a la ubicación, conlleva el problema de incluir a los estudiantes con discapacidades moderadas y graves en aulas regulares, en donde es menos probable que accedan a recursos e instalaciones especializadas, o canalizarlos a la educación especial, con el riesgo de negarles su derecho 
a la educación (Norwich, 2008a). En las personas con discapacidades más severas, esta situación cobra más relevancia porque en las escuelas regulares no hay docentes especializados, los recursos son escasos y los grupos tienden a ser numerosos (Norwich, 1993).

En cualquiera de los tres dilemas la solución no es sencilla ni definitiva, ya que lo que está de por medio no sólo es el lugar que ocupan los actores educativos, ni tampoco es un asunto que se reduzca únicamente a la disponibilidad de los recursos o a la cuestión de quién toma las decisiones educativas. Por supuesto todo esto entra juego, pero lo más importante es el conflicto entre los valores que subyacen a nuestras ideas sobre la diferencia. En suma, para Norwich (2008b) el dilema básico de la inclusión es "reconocer o no las diferencias, ya que cualquiera de estas opciones tiene implicaciones negativas o riesgos asociados con el estigma, la devaluación, el rechazo o la negación de oportunidades" (Norwich, 2008b: 287) (traducción propia).

\section{EL DILEMA DE LA INCLUSIÓN EN LA EDUCACIÓN SUPERIOR}

El dilema de la inclusión de las personas con discapacidad tiene su origen en la forma en que entendemos la diferencia; se remite a rasgos o comportamientos socialmente estigmatizados y está presente en todos los niveles educativos. Sin embargo, hasta ahora la mayoría de los trabajos sobre el tema se ha enfocado en la educación básica; en el nivel superior los dilemas han sido abordados de manera colateral, como parte de investigaciones más amplias referidas a las experiencias de los alumnos con discapacidad o de los docentes que han trabajado con ellos, pero no como objeto de estudio propiamente dicho.

En los siguientes apartados presentamos tres formulaciones de las disyuntivas que la diferencia produce en la educación superior: el dilema del mérito, el de la vocación y el de los ajustes. Ésta es una categorización que hemos construido a partir de los diferentes hallazgos de una investigación sobre inclusión de estudiantes con discapacidad en universidades mexicanas (Pérez-Castro, 2018; 2019a; 2019b; 2021). Como ya hemos dicho, Norwich (2008a) distingue entre el dilema de identificación, el del currículo y el de la ubicación. Si bien éstos también pueden encontrarse en la formación terciaria y los hemos trabajado previamente (Iturbide y Pérez-Castro, 2020), sostenemos que la educación universitaria tiene condiciones que necesitan ser abordadas en su especificidad.

Lo que a continuación exponemos forma parte del proceso de construcción del marco teórico de nuestra investigación; en particular, nos interesa nombrar y sustentar las situaciones dilemáticas discutidas en trabajos anteriores. No entraremos en el debate sobre los fundamentos filosóficos, antropológicos, sociológicos o históricos que intervienen en el dilema de la diferencia, algo que rebasaría los fines de este artículo y que además ha sido ampliamente discutido por los autores aquí revisados, principalmente Norwich (2008a; 2008b; 2013) y Minow (1990), sino que buscamos poner a consideración las posibilidades analíticas de las categorías que proponemos.

Para ello, retomamos los aportes que Dyson y Millward, Artiles y Norwich hacen sobre los dilemas, así como los resultados de investigaciones en donde se identifican problemáticas relacionadas con la inclusión en la educación superior, y los articulamos con las condiciones en las que operan las instituciones para acercarnos a algunas de las cuestiones que se encuentran en el centro de estas tres formulaciones: el mérito, la vocación y los ajustes.

\section{El dilema del mérito}

La educación superior constituye la cúspide de los sistemas de enseñanza; en ella se han formado gran parte de los críticos, pensadores sociales, políticos, funcionarios públicos, profesionistas, técnicos y todos aquellos que han buscado aportar algo a la sociedad, así como tener posibilidades de movilidad en ella 
(Brunner, 2002). Con el tiempo, la educación terciaria - y en particular las universidadesse atribuyeron el monopolio de la generación y trasmisión de los conocimientos y, aunque han surgido otros agentes que también desarrollan estas funciones, dichas instituciones continúan teniendo un lugar privilegiado tanto en los sistemas educativos como en el imaginario social (Altbach et al., 2009).

Sin embargo, a la par de lo anterior - $\mathrm{O}$ quizá como resultado de ello- la educación superior se ha distinguido por su carácter elitista y por la importancia otorgada al mérito individual. De este modo, aunque en los últimos 50 años la población estudiantil mundial se cuadriplicó, la tasa bruta de matriculación hasta 2018 era de 38 por ciento (UNESCO, 2020), ${ }^{2}$ lo que significa que escasamente dos quintas partes de las personas del grupo de edad correspondiente logran entrar a este nivel educativo. La proporción de estudiantes que ingresa a la educación terciaria ha sido históricamente baja; su democratización inició hacia la segunda mitad del siglo XX, aunque con marcadas diferencias entre los países (Brunner, 2002; Rama, 2009). Este proceso amplió las oportunidades de acceso a grupos que frecuentemente habían sido excluidos o cuya presencia era muy baja, como las mujeres, los hijos de obreros o trabajadores asalariados y personas con ocupación remunerada que querían continuar con los estudios universitarios (Gibbons, 1998; Rodríguez, 1998).

Pero, si bien la expansión favoreció a la diversificación del perfil de los estudiantes, esto no significó el ingreso para todos, al contrario, la educación superior siguió siendo un bien social al alcance de ciertos segmentos de la población. Las personas con discapacidad son uno de los colectivos que no ha logrado beneficiarse de este impulso democratizador. En primer lugar, porque su presencia en la matrícula a lo largo del sistema educativo es exigua: a pesar del fortalecimiento del enfoque de la inclusión, en muchos países la educación especial continúa siendo la principal o única opción formativa para estos individuos, o bien, cuando logran ingresar a la enseñanza regular, enfrentan un sinnúmero de barreras que terminan por excluirlos (OMS, 2011). En segundo lugar, porque en la oferta educativa para las personas con discapacidad después del nivel básico se siguen priorizando las opciones terminales, como el aprendizaje de oficios o actividades manuales, lo que clausura sus oportunidades de continuar con los estudios universitarios (Torres, 2014). Y, en tercer lugar, porque socialmente aún predomina una idea deficitaria y monolítica de la discapacidad que, de entrada, califica a estos sujetos desde sus carencias e invalida las diferencias que existen entre ellos en términos de aptitudes, habilidades, conocimientos e intereses.

De este modo, cuando llegan a la educación superior, independientemente del tipo de discapacidad se les etiqueta como incapaces y problemáticos, porque la atención se centra en lo que no pueden hacer y en la inversión o esfuerzo que le representará a la institución responder a sus necesidades (Dyson y Milward, 2000). Las personas con discapacidad regularmente requieren de apoyos específicos para cumplir con las exigencias de este nivel educativo, lo que acarrea dudas sobre sus méritos y talentos. Es decir, hay una especie de "elitismo académico... que percibe a los estudiantes con discapacidad como menos capaces intelectualmente y también con menos probabilidades de tener un buen desempeño, al igual que aquellos que actualmente conforman la norma" (Madriaga et al., 2011: 902) (traducción propia).

El dilema radica, entonces, en la posibilidad de valorar de manera adecuada los conocimientos y habilidades de estos alumnos y, al mismo tiempo, brindarles condiciones que les ayuden a superar las barreras institucionales. En la mayoría de las modalidades educativas el desempeño académico, usualmente medido a través de un examen, es la llave que determina el ingreso de los estudiantes (Chain

${ }^{2}$ Consultar: http://data.uis.unesco.org/index.aspx?queryid=142 (consulta: 10 de septiembre de 2020). 
et al., 2003; Tinklin y Hall, 1999). De este modo, sin importar si se trata de una persona con o sin discapacidad, o del tipo de discapacidad que tenga, los parámetros son los mismos, es decir, todos presentan la misma prueba, a todos se les da el mismo tiempo para contestarla $\mathrm{y}$ todos tienen que cumplir con los puntajes establecidos, si bien en algunas instituciones se han implementado apoyos, como los asistentes de lectura y escritura, la impresión del examen en Braille o la ampliación del tiempo asignado, además de la variación de los puntajes dependiendo de la carrera elegida. A pesar de esto, la prueba escrita sigue siendo la estrategia privilegiada, lo que pone en desventaja a los estudiantes con discapacidad, no porque no tengan las competencias académicas requeridas, sino porque el procedimiento de selección no es el más adecuado para ellos (Hanafin et al., 2007).

Otras instituciones establecen como prerrequisito un promedio mínimo de la secundaria superior, media superior para el caso de México, o bien, un puntaje acumulado a partir de exámenes que se realizan al concluirla (Scanlon et al., 2015). En cualquiera de los dos casos, los jóvenes entran en una ardua competencia que ganan no sólo los que alcanzan las mejores calificaciones, sino también aquéllos que tienen un conocimiento acumulado de lo que el sistema educativo espera de ellos. Las personas con discapacidad tienen menos oportunidades de hacerlo porque, por lo general, ingresan más tarde a las instituciones educativas, llevan trayectorias escolares discontinuas, muestran tasas más altas de deserción o ausentismo y, aunque cada vez es más frecuente encontrar personas que han hecho toda su formación en la educación regular, aún hay muchas que cursan una parte en el sistema especial y otra en el regular (OMS, 2011; Tinklin y Hall, 1999). La pregunta es si determinar los mismos estándares para todos los estudiantes es justo para las personas con discapacidad y, de no ser así, qué estrategias contribuirían a compensar sus desventajas.
En el otro extremo están las instituciones que valoran la capacidad del alumno para manejar las dificultades que puede encontrar a lo largo de la educación universitaria por encima de las calificaciones académicas (Borland y James, 1999). Aunque son menos frecuentes, en estas universidades se procura tener un equipo multidisciplinario para evaluar las competencias del estudiante para desenvolverse de manera autónoma y, al mismo tiempo, prever los apoyos que puede requerir a lo largo de sus estudios; sin embargo, esto conlleva varias dificultades porque cada tipo de discapacidad marca diferencias importantes en el desempeño de las personas, incluso teniendo la misma. Otra limitación es la falta de personal calificado para hacer estas evaluaciones, así como la ausencia de programas a largo plazo que respondan adecuadamente a las necesidades cambiantes de los alumnos (Wilson et al., 2000). Además, el problema es que estas medidas tienden a ser interpretadas como ventajosas para las personas con discapacidad y, por consiguiente, injustas para el resto de los aspirantes que compiten por un lugar en la institución (Madriaga et al., 2011).

En suma, como sostiene Dubet (2005), la crueldad del mérito es que nos ha hecho creer que el desempeño de los alumnos es únicamente producto de sus capacidades, su trabajo y su libertad. Además, se ha convertido en una ficción tan necesaria para el funcionamiento del sistema educativo, que esperamos que siempre opere de la misma manera y con los mismos criterios para todos. Cuando las reglas se modifican a favor de alguna persona o grupo lo consideramos injusto, pero cuando el individuo se esfuerza y no tiene éxito, entonces se le culpa de su propio fracaso: "todo sucede como si él hubiera decidido 'libremente' acerca de sus desempeños escolares, trabajando en mayor o menor medida...", de manera que terminamos aceptando que "...en realidad, él es diferente, menos dotado, menos valiente, menos eficiente" (Dubet, 2005: 33). He ahí el trasfondo del dilema del mérito. 


\section{El dilema de la vocación}

De acuerdo con Artiles (1988), la baja proporción de personas con discapacidad en la enseñanza regular se debe a que hemos entendido la diferencia como algo negativo, desagradable o desventajoso. Consecuentemente, solemos creer que estos alumnos no tienen el perfil para ser incluidos en las instituciones educativas (Artiles, 2003) y, peor aún, que nunca serán capaces de alcanzarlo. Si bien las deficiencias pueden marcar límites a la actuación de las personas, cabe recordar que, en primer lugar, la relación entre deficiencia y discapacidad no es lineal, sino que en esta última intervienen las barreras sociales que son las que, al interactuar con las capacidades individuales, terminan por crear entornos discapacitantes (Shakespeare, 2006). Y, en segundo lugar, no podemos generalizar sobre el desempeño académico de estas personas porque cada una posee distintos conocimientos, habilidades y competencias, al igual que ocurre con el resto de los individuos.

En la educación superior, la perspectiva deficitaria sobre la discapacidad se concreta, en buena medida, en lo que hemos denominado el dilema de la vocación. Cuando una persona con esta condición ingresa a la universidad se tiende a orientarla hacia disciplinas que no impliquen un gran esfuerzo físico o mucha movilidad, que tienen menos demanda o, incluso, que no exigen puntajes altos en la prueba de admisión. Lo anterior está en correlación con la exclusión que por mucho tiempo ha enfrentado la población con discapacidad; como ya decíamos, aún hoy, muchos de ellos son segregados a la educación especial y, en la peor de las circunstancias, encerrados en sus casas por el estigma que representan para sus familias. De este modo, pocas veces se espera que puedan llegar al nivel superior $\mathrm{y}$, cuando lo hacen, las instituciones no siempre están preparadas o dispuestas a recibirlos.

Para Moola (2015), esta actitud hace que de antemano muchas opciones vocacionales estén cerradas para ellos, en especial carreras liberales y con un alto prestigio, como medicina o ingeniería. Como ejemplo, relata la actitud de un profesor de psicología de la Universidad de Manitoba, para quien los estudiantes con discapacidad no tenían los elementos necesarios para ejercer esta profesión. Esta experiencia fue recuperada de un alumno con discapacidad intelectual, situación que seguramente influyó en el juicio del docente. Aquí, la pregunta es si tener ésta, o cualquier otra discapacidad tendría que ser un impedimento para ingresar a determinados programas.

Otro ejemplo es el expuesto por Ryan (2011) a partir de una investigación realizada en Australia con una muestra que incluyó oficiales de discapacidad, ${ }^{3}$ estudiantes, profesores y profesionales de enfermería. Los resultados, aunque no concluyentes, indicaron que los docentes constituían el grupo con mayor tendencia a excluir a las personas con discapacidad, principalmente aquéllas con problemas psiquiátricos, dislexia, enfermedades cardíacas, limitaciones para levantar pacientes y discapacidad visual. Para la autora, detrás de esto se encuentra la idea de "capacitismo", fuertemente institucionalizada en los establecimientos educativos.

Los supuestos normativos sobre el "enfermero ideal” influyen en las decisiones sobre quién debería ser parte de los cursos de pregrado en enfermería, ya que enfatizan fuertemente las nociones de aptitud física y consideran que la enfermedad, la discapacidad y la fragilidad física son condiciones que necesitan ser corregidas (Ryan, 2011: 80) (traducción propia).

\footnotetext{
El oficial de discapacidad es un tipo de funcionario universitario que funge como representante de los estudiantes con discapacidad en la universidad. También se encarga de realizar campañas de sensibilización, eventos académicos o cualquier otra actividad que contribuya a la trayectoria académica y la inclusión de estos alumnos. La figura del disability officer opera en universidades del Reino Unido, Canadá y Australia, principalmente. Hasta donde la investigación ha evidenciado, no hay un equivalente en las universidades de América Latina. El nombre del puesto es tal cual se tradujo: disability officer o university disability officer.
} 
Lo anterior se refleja en una diversidad de estructuras y normativas institucionales, así como de prácticas y actitudes de los actores educativos que operan de manera velada la mayoría de las veces, pero que a la larga resultan ser bastante efectivos, porque no sólo determinan quién puede y quién no puede ingresar a la educación superior, sino cuáles son las carreras más adecuadas de acuerdo con sus capacidades.

En contraste, en los programas de humanidades y ciencias sociales parece haber una mayor aceptación hacia estos estudiantes porque se cree que, debido a sus limitaciones, pueden "encajar" más fácilmente, o tendrán más oportunidades para concluirlas (Avendaño y Díaz, 2014). Adicionalmente, este tipo de carreras no siempre son muy valoradas, porque de manera reiterada se cuestiona su utilidad práctica, la ausencia de un nicho laboral específico y su poca contribución al desarrollo económico (Krotz, 2009). Incluso, se consideran opciones para aquellas personas que no tienen habilidades para campos más "científicos” (Trusz, 2020). Esta imagen se encuentra muy arraigada no sólo en las instituciones, sino en los padres familia y, a veces, en las propias personas con discapacidad que, por inseguridad respecto de su formación académica, así como de sus competencias individuales, terminan por registrarse en estos programas aunque no tengan vocación para ello.

En el otro extremo están aquellas posturas que cuestionan la pertinencia de que estas personas estudien carreras con estándares académicos altos o en las que está de por medio la seguridad de los usuarios o clientes. Distintas investigaciones realizadas con profesores y estudiantes universitarios (Santos y Lorenzo, 2010; Suriá et al., 2011) han señalado que en licenciaturas como Arquitectura y Biología, así como en programas de corte tecnológico, hay una actitud menos receptiva hacia los alumnos con discapacidad; esta resistencia es originada por las dudas sobre su autonomía y capacidad académica.
Lo anterior es especialmente notorio en las áreas de ciencias de la salud y de la docencia. En las primeras, el principal argumento es la integridad de los pacientes, ya que se supone que la actuación profesional de un enfermero o médico con discapacidad será menos eficiente que la de sus pares sin discapacidad (Ryan, 2011). Otros argumentos aluden a los estrictos estándares técnicos a los que deben sujetarse los médicos y a la capacidad que deben tener, tanto para trabajar en equipo, como de manera independiente. Adicionalmente, se ha señalado que, en el caso de la implementación de ajustes para incluir a los profesionales con discapacidad, se tendrían que valorar sus impactos en la seguridad de los pacientes y en el trato confidencial que debe asegurárseles. Por su parte, los alumnos con discapacidad han denunciado el constante escrutinio sobre su condición y desempeño (lo que no ocurre con sus pares sin discapacidad) y que a la postre desalienta sus aspiraciones profesionales (Meeks et al., 2015).

En el campo de la enseñanza las objeciones apuntan hacia la pérdida de autoridad frente a los colegas, padres de familia y alumnos, es decir, se asume que un docente con discapacidad no puede enseñar como el resto de sus compañeros, o bien, que su condición puede ser un impedimento para mantener el control del grupo. Ésta es una imagen muy común no sólo en la educación básica, sino también en la superior. En la revisión hecha por Neca et al. (2020: 8) se encontró que "tener una discapacidad se percibe como una barrera para emprender una carrera profesional en el mundo académico" (traducción propia); las actitudes negativas de sus compañeros, las dificultades para que los demás reconozcan su trabajo, los efectos en su autoestima y el temor a que sean encasillados en la educación especial son algunos de los obstáculos identificados por los maestros con discapacidad (Neca et al., 2020).

Desde otras perspectivas se admite que, si bien el estigma es la principal barrera para los docentes con esta condición, la situación para 
las instituciones educativas $-\mathrm{y}$ en especial para los directivos - no es tan sencilla porque tienen que adherirse a las disposiciones que, por ley, se han determinado para incluir a estas personas y al mismo tiempo atender a los requisitos de idoneidad profesional establecidos por el gremio (Sokal et al., 2017).

Por un lado, la homogeneidad que se asume entre los diferentes tipos de discapacidad puede ser utilizada de manera inapropiada para evitar que estos alumnos con discapacidad se gradúen de los programas de formación de maestros... Por otro lado, en sus prácticas, los estudiantes con discapacidad a veces exhiben ciertos comportamientos que llevan a preguntarse si su condición es incompatible con el cumplimiento de los estándares para el ejercicio profesional docente, incluso cuando se les brindan los ajustes necesarios (Sokal et al., 2017: 4) (traducción propia).

De este modo, en el dilema de la vocación hay una contraposición entre los principios profesionales, que marcan determinados estándares para la actuación profesional, así como para la protección de los usuarios o clientes; y los principios de justicia educativa, que demandan la igualdad en derechos, la participación plena y la equiparación de oportunidades para todos los estudiantes.

\section{El dilema de los ajustes}

Los ajustes son un elemento clave para la inclusión ya que permiten adaptar o modificar los contenidos, materiales y condiciones educativas para responder a las necesidades de los estudiantes y ampliar sus oportunidades de aprendizaje. Esto no es algo totalmente nuevo para los docentes, ya que a lo largo de los cursos suelen hacer adecuaciones con base en las pautas que les van dando los alumnos. Sin embargo, como advierten Dyson y Milward (2000), la diversidad que existe entre los miembros de una clase regular tiene determinados márgenes, o cuando menos eso es lo que se espera, de manera que cuando alguien se sale significativamente de ellos se originan tensiones, tanto para los docentes y las instituciones, como para los estudiantes.

El tercer dilema que queremos abordar en la educación superior es el que se refiere a los ajustes para los alumnos con discapacidad, los cuales, por una parte, acarrean dudas sobre las posibles ventajas que pueden representar para ellos, especialmente cuando se les compara con las exigencias a las que se acogen sus demás compañeros y, por otra, terminan por reforzar la imagen negativa sobre la discapacidad.

El tema de los ajustes no siempre es sencillo porque abarca distintos niveles del trabajo institucional, desde las instalaciones físicas y los trámites administrativos hasta los contenidos curriculares y las actividades extraclases. Su implementación demanda que el personal, especialmente profesores y directivos, cuente con una base que le permita distinguir la pertinencia y el impacto de los diferentes ajustes, así como ser receptivos a las necesidades de los estudiantes. Por su parte, las personas con discapacidad deben estar conscientes de que no son meros receptores de los ajustes, sino que tienen un rol activo en su gestión y posibles alcances.

Asimismo, en la implementación se pueden suscitar algunos conflictos entre los profesores y los estudiantes con y sin discapacidad, porque las modificaciones o adaptaciones dirigidas a determinados individuos llegan a interpretarse como privilegios que atentan contra la igualdad de condiciones que deben mantener las instituciones. Al respecto, en una investigación hecha con docentes universitarios Moriña y Carballo (2018) muestran que éstos frecuentemente se sienten presionados no sólo porque desconocen qué tipo de ajustes son los más adecuados en cada caso y cómo realizarlos, sino por el riesgo de "discriminar al alumno, tanto por defecto como por exceso" (Moriña y Carballo, 2018: 90). López (2019) presenta otro ejemplo en donde, a partir de la solicitud de un estudiante con 
discapacidad se organizó un seguimiento con los docentes que le impartían clases, pero esto provocó molestia entre sus compañeros porque, desde su punto de vista, se le estaba dando una atención preferencial. Al final, el coordinador de la carrera decidió no continuar con la estrategia, ni para este alumno ni para ningún otro, porque asumía que "lejos de ayudarlo, señalaba su condición de desventaja y podría predisponer a los profesores hacia el joven con discapacidad" (López, 2019: 132).

Lo anterior nos remite claramente al razonamiento inicial de Minow (1990), es decir, ¿hasta dónde tratar diferencialmente a las personas con discapacidad acentúa sus desventajas y hasta dónde tratarlas de manera similar nos hace insensibles ante ellas? Lo peor es que, como advierte Goffman (1970), el individuo etiquetado como "diferente" es consciente del malestar que produce en los otros, que intentan mediar entre el interés excesivo y la indiferencia de su condición; mientras que los otros, considerados a sí mismos como "normales", también se dan cuenta de que el estigmatizado se percata de lo difícil de la situación.

En los estudiantes con discapacidad se ha observado que constantemente luchan por no ser encasillados como "especiales", "dependientes" o "débiles", al tiempo que reconocen que solicitar o recibir ciertos apoyos incrementa el riesgo de que se les asocie con este tipo de adjetivos. Como denuncia Moola (2015: 49), los ajustes pueden convertirse en una espada de doble filo porque si bien "facilitan el aprendizaje, pueden 'marcar y estigmatizar' a los estudiantes con discapacidad haciéndolos hipervisibles. La dificultad para realizar los ajustes puede estar relacionada con la falta de conciencia sobre la discapacidad en el propio campus" (traducción propia). En algunos casos, los estudiantes deciden no pedir ningún tipo de apoyo a fin de no ser tratados de manera condescendiente (Madriaga, 2007), o para no ocasionar problemas o resquemores entre sus compañeros, especialmente cuando están de por medio las calificaciones (Pérez-Castro,
2018). Es decir, el dilema de los ajustes no sólo atañe a los profesores, sino también a los estudiantes con discapacidad.

Esta disyuntiva permea hasta la dimensión institucional. En una investigación realizada en cuatro universidades, tres de América Latina y una en España, Torres (2014) subraya la preocupación de algunas autoridades institucionales por hacer notar que, a todos los alumnos, con o sin discapacidad, se les da el mismo trato y que en ningún momento se realizan acciones que puedan convertirse en ventajas de unos frente a otros. De este modo, los ajustes que se ofrecen son bastante acotados, usualmente referidos a adaptaciones físicas. Incluso, se llega a plantear que esta medida no sólo ayuda a evitar los sesgos hacia los estudiantes con discapacidad, sino que además es una manera para que ellos demuestren que realmente se han "ganado" su lugar en la universidad.

Lo anterior constituye un falso dilema, porque preservar la igualdad de oportunidades no debería significar desconocer las diferencias entre los estudiantes; al contrario, implicaría reconocerlas para tomar medidas que hagan que todos se sientan incluidos. No obstante, como ya hemos dicho, los dilemas son elaboraciones que las personas hacen a partir de la situación o condiciones en las que se encuentran (Banks y Williams, 2005). En esta tercera formulación del dilema de la inclusión, el poco conocimiento y empatía sobre las capacidades y necesidades de los alumnos con discapacidad, así como las carencias formativas respecto a la inclusión o sobre las estrategias y recursos que se pueden aprovechar - tanto a nivel de la clase como de la institución-son factores que concurren en su configuración.

\section{CONSIDERACIONES FINALES}

Los dilemas son situaciones problemáticas que nos confrontan con la toma de decisiones y en las que los sujetos consideran que hay un costo o pérdida moral para todos los 
involucrados. Su naturaleza irresoluble nos genera incertidumbre e incomodidad, porque siempre existe la duda respecto al curso de acción que tomamos y las posibles consecuencias de haberlo hecho de otra manera. Sin embargo, justamente en este carácter problemático es en donde, creemos, reside la potencialidad de los dilemas, ya que nos permiten reflexionar sobre lo que hacemos y sobre la forma en que podríamos mejorarlo.

Específicamente, en la inclusión de los estudiantes con discapacidad en educación superior se conjugan múltiples ámbitos, por ejemplo, en el dilema del mérito se empalman temas pedagógicos sobre cómo evaluar adecuadamente los conocimientos y habilidades de estos alumnos, con cuestiones de orden filosófico sobre la justicia social, la equiparación de oportunidades y la construcción social del mérito. En el dilema de la vocación están presentes, por un lado, las aspiraciones de justicia educativa, que apuntan a la disminución de la brecha entre los más y los menos favorecidos $y$, por otro, los principios que regulan el buen ejercicio profesional y salvaguardan la integridad de los usuarios o clientes. Y, por último, en el dilema de los ajustes intervienen asuntos relacionados con la formación de los profesores respecto a las capacidades y necesidades de los alumnos, la sensibilización y conocimiento que los estudiantes sin discapacidad tienen de sus pares con discapacidad y la flexibilidad del régimen académico para hacer accesibles los distintos procesos institucionales.

Estas tres formulaciones dilemáticas no son las únicas que se enfrentan en la educación superior; por el contrario, a lo largo de la dinámica de las clases, las relaciones interpersonales y la participación en la vida institucional, muy probablemente profesores y estudiantes tienen que lidiar con otro tipo de situaciones problemáticas que los llevan a elegir entre la inclusión y la separación, la neutralidad y la acomodación, el trato similar y el trato especial (Minow, 1990). La inclusión educativa en muchos sentidos se torna problemática para los actores educativos por los inconvenientes prácticos que enfrentan cotidianamente, ya que, aunque cada vez hay un mayor conocimiento sobre ella, lo cierto es que las instituciones no siempre están suficientemente preparadas para atender las necesidades de los alumnos con discapacidad. Las razones son diversas, en primer lugar, el sistema educativo mantiene y reproduce múltiples barreras estructurales, pedagógicas, físicas y actitudinales que incrementan el riesgo de exclusión de estas personas; en segundo, la población con discapacidad ha sido una de las últimas en beneficiarse de la ampliación de oportunidades en el nivel superior; $y$, en tercero, los establecimientos educativos tienen pautas de actuación organizadas para responder a la "normalidad", de manera que alguien que no encaja en este modelo, con o sin discapacidad, tiene muchas dificultades para cumplir con los requisitos y estándares académicos.

El contraste entre lo que se plantea a nivel de la normatividad y las políticas educativas, en donde hay una creciente presencia del enfoque de la inclusión, y las condiciones reales con las que operan las instituciones, es el trasfondo que da pie al surgimiento de los dilemas. Por otra parte, aunque, como advierten los especialistas en el tema, no existen soluciones definitivas para estas disyuntivas, esto no significa que las posibilidades para la intervención y el cambio estén cerradas. Por ejemplo, en el tema del mérito, como ya mencionábamos, algunas instituciones han buscado abrir espacios para la discusión y desarrollar estrategias que permitan valorar los conocimientos y habilidades de las personas con discapacidad. Por supuesto, lo anterior trae nuevas interrogantes respecto a la pertinencia de estas propuestas en términos de tiempo y recursos materiales, la formación del personal que estará a cargo de su implementación, así como el logro de un equilibrio entre las exigencias académicas y la equidad. A pesar de esto, los dilemas hacen que los actores educativos de alguna manera se sientan 
interpelados y ensayen diversas medidas, aunque estén conscientes de que los problemas no se superarán de una vez y para siempre.

Es decir, la complejidad de los dilemas no nos exime de enfrentarlos y, por el contrario, éstos nos permiten reconocer que su solución conlleva la participación de los diferentes actores involucrados. Los profesores o estudiantes en lo individual no pueden asumirse como los únicos responsables, sino que es necesario el trabajo coordinado de docentes, alumnos, directivos e, incluso, de la familia y organizaciones de la sociedad civil, como han hecho algunas universidades que han abierto su participación a los padres de los estudiantes y a organizaciones de personas con discapacidad o que atienden a esta población.

A la par de lo que hemos señalado, nos interesa insistir en que el dilema de la diferencia y sus posibles formulaciones no es un tema exclusivo de los estudiantes con discapacidad. En las instituciones educativas aún prevalecen prácticas, condiciones y normas que terminan por excluir a los que no se ajustan al "estudiante modelo". Esto es particularmente evidente en la educación superior, en donde predomina la idea del alumno de tiempo completo, clase media o alta y plenamente autónomo. Sin embargo, la ampliación de las oportunidades de acceso a lo largo del sistema educativo ha hecho que con mayor frecuencia lleguen a la universidad individuos que no cumplen con este perfil, como las personas que estudian y trabajan, las de origen indígena y las jefas de familia, entre otros. Estos estudiantes también enfrentan barreras para su permanencia, logro académico y egreso, lo que eventualmente se convierte en caldo de cultivo de situaciones dilemáticas que los afectan a ellos, a sus profesores y a la institución.

Finalmente, cabría recordar que los dilemas no son entidades inmutables, no sólo porque se trata de construcciones que los sujetos hacemos frente a determinadas circunstancias, sino porque sus contenidos cambian con el tiempo y el contexto en donde se presenten. Esto no significa caer en el relativismo; de hecho, los dilemas parten de problemas reales y tienen efectos muy concretos en la vida de las personas; es por ello por lo que tenemos que estar mejor preparados, comprometidos con nuestro trabajo en las instituciones educativas y abiertos para vislumbrar los cursos de acción que nos plantean estas disyuntivas, así como sus posibles consecuencias.

\section{REFERENCIAS}

Allen, Karen (2012), "What is an Ethical Dilemma?", The New Social Worker, vol. 19, núm. 2, pp. 4-6.

Altbach, Philip, Liz Reisberg y Laura Rumbley (2009), Trends in Global Higher Education: Tracking an academic revolution, París, UNESCO.

Artiles, Alfredo (1998), "The Dilemma of Difference: Enriching the disproportionality discourse with theory and context", The Journal of Special Education, vol. 30, núm. 1, pp. 32-36.

Artiles, Alfredo (2003), "Special Education's Changing Identity: Paradoxes and dilemmas in views of culture and space", Harvard Educational Review, vol. 73, núm. 2, pp. 164-202.
Avendaño, Cecilia y Jessica Díaz (2014), “El proceso de integración educativa desde la perspectiva de cinco jóvenes universitarios con discapacidad visual de Concepción”, Estudios Pedagógicos, vol. 40, núm. 2, pp. 45-64.

BANks, Sarah y Robin Williams (2005), "Accounting for Ethical Difficulties in Social Welfare Work: Issues, problems and dilemmas", British Journal of Social Work, vol. 35, núm. 7, pp. 1005-1022.

Borland, John y Sue James (1999), “The Learning Experience of Students with Disabilities in Higher Education. A case study of a UK University”, Disability \& Society, vol. 14, núm. 1, pp. 85-101.

BRunNer, José (2002), “Educación superior: desafíos y tareas”, Realidad y Reflexión, núm. 4, pp. 61-81. 
Chain, Ragueb, Nicandro Cruz, Manuel Martínez y Nancy Jácome (2003), “Examen de selección y probabilidad de éxito escolar en estudios superiores. Estudio en una universidad pública estatal mexicana", Revista Electrónica de Investigación Educativa, vol. 5, núm. 1, pp. 100-116.

Coulon, Alain (1995), Ethnomethodology. Qualitative research methods, Thousand Oaks, Sage Publications.

Dubet, François (2005), La escuela de las oportunidades ¿Qué es una escuela justa?, Barcelona, Gedisa.

Dyson, Allan (2001), "L'equitat com al camí a l'excellència? Possibilitats i reptes en l'educació inclusiva", Revista de Psicologia, Ciències de l'Educació $i$ de l'Esport, núm. 5, pp. 91-106.

Dyson, Allan y Allan Millward (2000), Schools and Special Needs. Issues of innovation and inclusion, Londres, Sage Publications.

Gibbons, Michael (1998), Pertinencia de la educación superior en el siglo XXI, Washington, Banco Mundial.

Goffman, Erving (2001 [1970]), Estigma: la identidad deteriorada, Madrid, Amorrortu.

Hanafin, Joan, Michael Shevlin, Mairin Kenny y Eileen McNeela (2007), "Including Young People with Disabilities. Assessment challenges in higher education", Higher Education, vol. 54, núm. 3, pp. 435-448, en: https://www. researchgate.net/publication/226230738_Including_young_people_with_disabilities_ Assessment_challenges_in_higher_education (consulta: 22 de septiembre de 2020).

Iturbide, Paulina y Judith Pérez-Castro (2020), "Dilemas del profesorado en la inclusión educativa de estudiantes universitarios con discapacidad", Revista de Investigación Educativa de la REDIECH, vol. 11, núm. E1007, pp. 1-21. DOI: https://doi.org/10.33010/ie_rie_rediech. v11i0.1007

Judge, Harry (1981), “Dilemmas in Education”, Journal of Child Psychology and Psychiatrics, vol. 22, núm. 2, pp. 111-116.

Krotz, Esteban (2009), "Hacia la recuperación del lugar de las ciencias sociales en la sociedad de conocimiento", Revista Mexicana de Sociología, vol. 71, número especial, pp. 75-104.

López, Alicia (2019), “Accesibilidad académica en educación superior", en Judith Pérez-Castro y Alicia López (coords.), Discapacidad, inclusión social y educación, México, UNAMIISUE, pp. 125-141.

Lowe, Eugene (1999), "Promise and Dilemma: Incorporating, racial diversity in selective higher education", en Eugene Lowe (ed.), Promise and Dilemma: Perspectives on racial diversity and higher education, Princeton, Princeton University Press, pp. 3-44.
MadriagA, Manuel (2007), "Enduring Disablism with Dyslexia and their Pathways into UK Higher Education and Beyond", Disability \& Society, vol. 22, núm. 4, pp. 399-412.

Madriaga, Manuel, Katie Hanson, Helen Kay y Ana Walker (2011), "Marking-out Normalcy and Disability in Higher Education", British Journal of Sociology of Education, vol. 32, núm. 6, pp. 901-920.

Meeks, Lisa, Joan Bisagno, Neera Jain y Kurt Herzer (2015), "Support Students with Disabilities in Medicine and Health Care Programs", Disability Compliance for Higher Education, vol. 21, núm. 3, pp. 1-5.

Minow, Martha (1990), Making all the Difference, Ithaca, Cornell University Press.

Moola, Fiona (2015), “The Road to the Ivory Tower: The learning experiences of students with disabilities at the University of Manitoba", Qualitative Research in Education, vol. 4, núm. 1, pp. 45-70.

MoriÑA, Anabel y Rafael Carballo (2018), "Profesorado universitario y educación inclusiva: respondiendo a sus necesidades de formación", Psicologia Escolar e Educacional, número especial, pp. 87-95.

NeCA, Patrícia, Maria Borges y Paula Campos (2020), "Teachers with Disabilities: A literature review", International Journal of Inclusive Education, pp. 1-19. DOI: https://doi.org/10.1080/1 3603116.2020.1776779

Norwich, Brahm (1993), "Ideological Dilemmas in Special Needs Education: Practitioners' views", Oxford Review of Education, vol. 19, núm. 4, pp. 527-546.

Norwich, Brahm (2008a), Dilemmas of Difference Inclusion and Disability. International perspectives and future directions, Abingdon, Routledge.

Norwich, Brahm (2008b), "Dilemmas of Difference, Inclusion and Disability: International perspectives on placement", European Journal of Special Needs Education, vol. 23, núm. 4, pp. 287-304.

Norwich, Brahm (2013), Addressing Tensions and Dilemmas in Inclusive Education. Living with uncertainty, Abingdon, Routledge.

Norwich, Brahm (2014), "Recognising Value Tensions that Underlie Problems in Inclusive Education", Cambridge Journal of Education, vol. 44, núm. 4, pp. 495-510.

OMS (2011), Informe mundial sobre discapacidad, Ginebra, OMS.

Parrilla, Ángeles (2002), “Acerca del origen y sentido de la educación inclusiva", Revista de Educación, núm. 327, pp. 11-29.

PÉrez-Castro, Judith (2018), “Condiciones para la inclusión de los estudiantes universitarios con discapacidad en el trabajo áulico", Memorias del Congreso Internacional de Investigación 
Academia Journals, Morelia, Academia Journals, pp. 2615-2621.

PÉrez-Castro, Judith (2019a), "Entre barreras y facilitadores: las experiencias de los estudiantes universitarios con discapacidad", Sinéctica, núm. 53, pp. 1-22, en: https://sinectica. iteso. $\mathrm{mx} /$ index.php/SINECTICA/article/ view/948/1077 (consulta: 1 de marzo de 2021).

PÉrez-Castro, Judith (2019b), "La inclusión de los estudiantes con discapacidad en dos universidades públicas mexicanas", Innovación Educativa, vol. 19, núm. 79, en: https://www. ipn.mx/assets/files/innovacion/docs/Innovacion-Educativa-79/La-inclusion-de-los-estudiantes-con-discapacidad-en-dos-universidades.pdf (consulta: 1 de marzo de 2021).

PÉrez-Castro, Judith (2021), "Condiciones para la docencia inclusiva: análisis desde las barreras y los facilitadores", Revista Iberoamericana de Educación Superior, vol. 12, núm. 33, pp. 138-157. DOI: https://doi.org/10.22201/ iisue.20072872e.2021.33.862

RAmA, Claudio (2009), "La tendencia a la masificación de la cobertura de la educación superior en América Latina", Revista Iberoamericana de Educación, vol. 50, pp. 173-195.

Rodríguez, Roberto (1998), "Expansión del sistema educativo superior en México 1970-1995”, en Magdalena Fresán (ed.), Tres décadas de políticas de Estado en la educación superior, México, ANUIES, pp. 167-205.

Ryan, Janette (2011), "Access and Participation in Higher Education of Students with Disabilities: Access to what?", Australian Educational Researcher, vol. 38, núm. 1, pp. 73-93.

SAntos, Miguel y María del Mar Lorenzo (2010), "Dimensión cívica y desarrollo formativo de los estudiantes universitarios en el contexto español", Revista Electrónica de Investigación Educativa, vol. 12, número especial, pp. 1-17, en: https://redie.uabc.mx/redie/article/view/250/411 (consulta: 8 de septiembre de 2020).

ScAnlon, Geraldine, Michael Shevlin y Conor McGuckin (2015), "(Dis)ability and Choice. The dilemmas of young people's transitions to further and higher education in Ireland", en Peter Kelly y Annelies Kamp (eds.), A Critical Youth Studies for the 21st Century, Leiden, Brill, pp. 105-122.
Shakespeare, Tom (2006), “The Social Model of Disability”, en Lennard Davis (ed.), The Disability Studies Reader, Nueva York, Routledge, pp. 197-204.

Skrtic, Thomas (1991), "The Special Education Paradox. Equity as the way to excellence", Harvard Educational Review, vol. 61, núm. 2, pp. 148-206.

SoKAL, Laura, Debra Woloshyn y Alina Wilson (2017), "Pre-service Teachers with Disabilities: Challenges and opportunities for directors of student teaching in Western Canada", The Canadian Journal for the Scholarship of Teaching and Learning, vol. 8, núm. 3, pp. 1-18, en: https://files.eric.ed.gov/fulltext/EJ1162955. pdf (consulta: 20 de septiembre de 2020).

Suriá, Raquel, Agustín Bueno y Ana María Rosser (2011), "Prejuicios entre los estudiantes hacia las personas con discapacidad: reflexiones a partir del caso de la Universidad de Alicante", Alternativas, núm. 18, pp. 75-90. DOI: https:// doi.org/10.14198/ALTERN2011.18.04

Tinklin, Teresa y John Hall (1999), "Getting Round Obstacles: Disabled students' experiences in higher education in Scotland", Studies in Higher Education, vol. 24, núm. 2, pp. 183-194.

Tomasevki, Katarina (2004), El asalto a la educación, Barcelona, Intermón Oxfam.

Torres, Zeferina (2014), Las entidades especializadas en los servicios a los estudiantes con discapacidad en cuatro universidades, un estudio comparativo, Tesis de Maestría, San Luis Potosí, El Colegio de San Luis (México), en: https://biblio.colsan.edu.mx/tesis/TorresCuevasZeferinaCatalina.pdf (consulta: 20 de septiembre de 2020).

Trusz, Slawomir (2020), "Why do Females Choose to Study Humanities or Social Sciences, while Males Prefer Technology or Science? Some intrapersonal and interpersonal predictors", Social Psychology of Education, núm. 23, pp. 615-639.

UNESCO (2017), Desglosar el objetivo de desarrollo sostenible 4: Educación 2030, París, UNESCO.

Wilson, Kristin, Elizabeth Getzel y Tracey Brown (2000), "Enhancing the Post-Secondary Campus Climate for Students with Disabilities", Journal of Vocational Rehabilitation, vol. 14, núm. 1, pp. 37-50. 